\title{
Agenesis of corpus callosum and emotional information processing in schizophrenia
}

\author{
Ovidiu Lungu ${ }^{1,2}$ and Emmanuel Stip ${ }^{1,3}$ * \\ ' Departement de Psychiatrie, Université de Montréal, Montréal, OC, Canada \\ ${ }^{2}$ Centre de Recherche de I'Institut Universitaire de Gériatrie de Montréal, Montréal, QC, Canada \\ ${ }^{3}$ Centre de Recherche Fernand Seguin, Affilié à I'Université de Montréal, Montréal, OC, Canada
}

\section{Edited by:}

Alex Fornito, University of Melbourne, Australia

\section{Reviewed by:}

Aristotle Voineskos, Centre for Addiction and Mental Health, Canada Tarek Rajji, Centre for Addiction and Mental Health, Canada

Mark Walterfang, Royal Melbourne Hospital, Australia

\section{*Correspondence:}

Emmanuel Stip, Centre de Recherche Centre Hospitalier de I'Université de Montréal, 1560, Sherbrooke East, Montreal, OC, Canada H2L 4M1. e-mail:emmanuel.stip@umontreal.ca
Corpus callosum (CC) is essential in providing the integration of information related to perception and action within a subcortico-cortical network, thus supporting the generation of a unified experience about and reaction to changes in the environment. Its role in schizophrenia is yet to be fully elucidated, but there is accumulating evidence that there could be differences between patients and healthy controls regarding the morphology and function of $\mathrm{CC}$, especially when individuals face emotionally laden information. Here, we report a case study of a patient with partial agenesis of corpus callosum (agCC patient with agenesis of the anterior aspect, above the genu) and we provide a direct comparison with a group of patients with no apparent callosal damage (CC group) regarding the brain activity during the processing of emotionally laden information. We found that although the visual cortex activation in response to visual stimuli regardless of their emotional content was comparable in agCC patient and CC group both in terms of localization and intensity of activation, we observed a very large, non-specific and non-lateralized cerebral activation in the agCC patient, in contrast with the $\mathrm{CC}$ group, which showed a more lateralized and spatially localized activation, when the emotional content of the stimuli was considered. Further analysis of brain activity in the regions obtained in the CC group revealed that the agCC patient actually had an opposite activation pattern relative to most participants with no CC agenesis, indicating a dysfunctional response to these kind of stimuli, consistent with the clinical presentation of this particular patient. Our results seem to give support to the disconnection hypothesis which posits that the core symptoms of schizophrenia are related to aberrant connectivity between distinct brain areas, especially when faced with emotional stimuli, a fact consistent with the clinical tableau of this particular patient.

Keywords: schizophrenia, agenesis, corpus callosum, fMRI

\section{INTRODUCTION}

The corpus callosum (CC), with its central position in the cerebrum, has an essential role in relaying sensory, motor, and cognitive information from homologous brain regions across cerebral hemispheres (de Lacoste et al., 1985; Buklina, 2005). A review of studies on patients with varying degrees of callosal damage (from complete agenesis, partial atrophies to subtle degradations), with and without interhemispheric disconnection, revealed that callosal functions are distributed and dissociated along an antero-posterior direction, with anterior callosal regions involved in interhemispheric inhibition in cognitive and perceptual (visuospatial) tasks requiring the management of resource competition (e.g., Stroop, hierarchical letters), and with posterior callosal areas implicated in interhemispheric facilitation processes at visuomotor and cognitive levels (Schulte and Muller-Oehring, 2011). Taken together, these findings argue for an important role of CC in the integration of information related to perception and action within a subcortico-cortical network, most likely supporting the generation of a unified experience about and reaction to changes in the environment.
The agenesis of corpus callosum (agCC) is defined as the absence, at birth, of parts or of the entire structure (National Institute of Neurological Disorders and Stroke, NIH). Studies comparing healthy individuals with those presenting the agCC found that highly functioning agCC patients have trouble processing emotional information (e.g., interpreting correctly the arousal generated by emotional stimuli; Paul et al., 2006) or a marked deficit in processing social information (Brown and Paul, 2000; Symington et al., 2011). In addition, a review of studies with agCC and autism spectrum disorder (ASD) subjects suggests that the theory-of-mind and emotion-processing deficits in agCC are similar to those observed in ASD (Booth et al., 2011). These findings suggest that CC plays an important role in processing of emotional information, especially in the social contexts, where the fast recognition of emotions seems to require interhemispheric cooperation, rather than hemispheric lateralization (Tamietto et al., 2007). While it is true that at the moment there are few studies comparing agCC individuals with healthy individuals (probably due to the low prevalence of this condition in the general population), recent techniques such as diffusion tension imaging could 
lead in the future to more studies with healthy individuals which would correlate social and emotional information processing with the structural integrity of this part of the brain.

Understanding the role of CC in schizophrenia has long been of interest since post-mortem anatomical morphology investigations suggested decreased thickness, cross-sectional area, and fiber density in these patients (Rosenthal and Bigelow, 1972; Bigelow et al., 1983; Highley et al., 1999). More recently, in vivo magnetic resonance imaging studies revealed that callosal area is reduced relative to healthy controls, more so in first-episode than in chronic patients (Arnone et al., 2008, for a review). However, despite these advances, the functional consequences stemming from these morphological differences in CC have not been systematically explored. Given the evidence that agCC was found to be associated with deficits in emotion recognition, labeling of emotional arousal, and decoding visual social cues even in highly functioning and otherwise healthy individuals (Paul et al., 2006; Symington et al., 2011), it is conceivable that callosal dysgenesis in schizophrenia may also be associated with dysfunction in the treatment of emotionally laden information. Two case studies of patients with schizophrenia and agCC provided such evidence (Hallak et al., 2007; Micoulaud-Franchi et al., 2011), but a direct comparison between these kind of individuals and schizophrenia patients with no evidence of callosal abnormality has not been attempted.

Here, we report a case study of a patient with partial agCC and we also provide a direct comparison with a group of patients with no evident callosal abnormality regarding the brain activity during processing of emotionally laden information.

\section{MATERIALS AND METHODS \\ PARTICIPANTS AND CLINICAL ASSESSMENT}

\section{Schizophrenia patient with agenesis of corpus callosum patient}

The patient of interest was a young female (23 years of age) diagnosed with schizophrenia 1 year before hospitalization (age 22) and discovered at the time as having agCC. Specifically, the dorsal
$\mathrm{CC}$ aspect, above the genu, and surrounding the midline was dysgenic or atrophied, including the istmus and anterior half of the spelnium; the Probst bundles were also atrophied. The lateral ventricles were large, especially in the posterior side, extending into the occipital lobe (up to $-82 \mathrm{~mm}$ posterior from the anterior commissure, in the Talairach space). For a comparison, we present in Figure 1 this patients' anatomical image normalized to Talairach template (Talairach and Tournoux, 1988), in the three planes (transversal, coronal, sagittal) side-by-side with the averaged anatomical image of the 13 schizophrenic patients with no CC agenesis, also normalized to Talairach template.

Personal history. The 23-year-old agCC patient was a single woman living with her brother and father. Her parents were separated; she was unemployed, living on social support. She was brought to the emergency department because she had bitten her father; in fact, for weeks she could not bear to be approached, or allowed anyone to enter into her personal space. She avoided contact with people for several months after the biting incident.

Clinical tableau. The patient had rituals of washing often her hands and clothes because of a fear of germs. She also collected objects, in particular issues from the magazine "People," predominantly for the performances of the American singer Mariah Carey. In addition to this, her environment was completely disordered. In fact she manifested a megalomaniacal delusion in regards to Maria Carey, the American singer, with whom she believed she had a very privileged relationship - a mental puerilism. In the emergency phase, we observed that the patient was obsessed with several ritual events and feelings of persecution and that she was capable of extreme anger, lacked judgment about her condition and did not understand that she can get medical help. Patient's persecution belief revolved around the idea that every person around her was conspiring against her, wishing her ill. Her solution to this situation was to move out and leave the city, to flee her persecutors and,

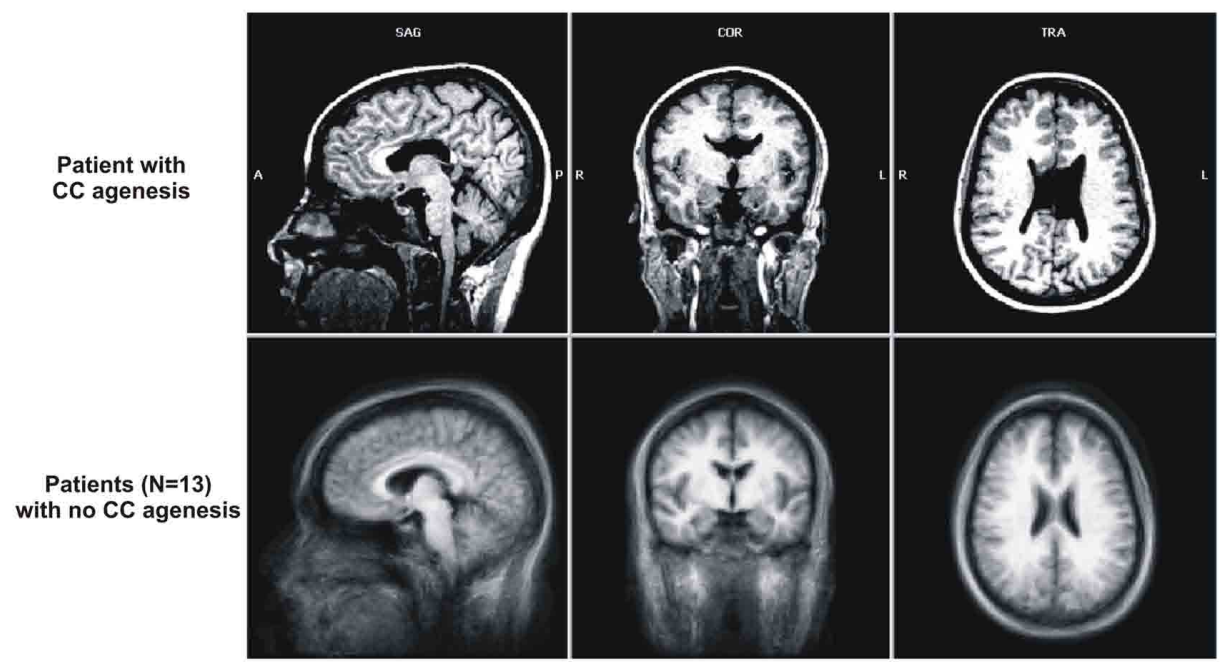

FIGURE 1 |The anatomical images for the schizophrenia patient with corpus callosum (CC) agenesis (top) and the average anatomical images for the 13 schizophrenia patients with no apparent $\mathbf{C C}$ agenesis. All images are normalized to Talairach space and the coordinates are Talairach coordinates. 
at last, to end up with Maria Carey, who - she believed - was waiting for her. In her mind, Maria Carey represented a confidante, a friend. She also experienced irritability, and there were recurrent refusals to take her antipsychotics or anxiolytics. From time to time, her mood became labile and she believed that her father wanted to destroy or even rape her. She had social difficulties and strained relationship with both her parents and, at the age of 15 , she thought that she was possessed by the devil. At times, she refused to wash her body, believing that this could endanger the life of the singer Maria Carey; in contrast she washed her hands obsessively. She manifested visual distortions as well as visual hallucinations.

Neuropsychological evaluation. The patient was right-handed, was able to write her name, and other phrases. Her drawings were accurate - a clock, a bicycle, a person. She was able to copy the position of her right arm or leg with the left, whilst her eyes were closed. She was able to draw a spiral with each hand separately and both together. Her head circumference was $56.7 \mathrm{~cm}$ (75th percentile). The patient presented no facial dimorphisms nor of the limbs, although the patient would not allow an inspection of her feet for reasons that she would not divulge. At the level of language, there was no dysphasia or lack of words. Overall intellectual performance was however at the lower end of average $(I Q=87)$. She completed the Wechsler Adult Intelligence Scale (Wechsler, 2008), the revised Wechsler test for memory and the Boston diagnostic aphasia examination. She performed the reading tests and tracking of letters and symbols, written numerical problems; drawings from Wechsler's memory scale, complex figures of Rey, logic of stories, California verbal learning test, left-right distinction, trail making A and $B$, generation of words and proverbs. She presented no visualperceptive or visuo-constructive difficulties and she appeared to have adequate visual coding, with no memory difficulties. However, encoding a list of 16 words in 5 tried revealed difficulties. We noted perseverations and retrospective interference and a notable delayed recall. Encoding of short stories is deficient. There were no attentional disorders in either selective or sustained attention. At the level of frontal functions, we noted a form of apathy in the generation of words, and a tendency to give up. Abstract thought measured by the interpretation of proverbs was clearly defective; there were many errors when performing the Stroop test. Examination of left-right distinction revealed difficulties in designating the right and left sides of another person when standing opposite her and we observed that the patient had to turn to be on the same side as the other person to arrive at the correct answer. Then there were also difficulties in double instructions. Examination of praxis revealed that the execution of symbolic gestures was adequate but the imitation of simple gestures was difficult to evaluate. We observed a tendency to laugh a lot during the testing and we noted that the gestures were still rough and performed incorrectly, which seems to suggest an ideomotor dyspraxia. Many bilateral mistakes were made in the examination of graph esthesia, but the patient did not present adiadochokinesia.

Psychiatric evaluation. Based on DSM-IV, the patient was diagnosed with a schizophreniform syndrome, with impulsivity and difficulty in anticipating the consequences of her behavior and planning difficulty. The Calgary depressions scale did not show a score of depression and her Positive and Negative Syndrome Scale (PANSS; Kay et al., 1987) total score was 83 (positive scale score: 23; negative scale score: 18). She had an air of listlessness and cognitive rigidity, which distinguished the obsessional component of this patient's symptoms and classic OCD, namely the egosyntonic aspect of her rituals which borders on delusion. As well as these atypical obsessional manifestations the patient seemed to show a deficiency in theory-of-mind, notably the incapacity to interpret the intentions and actions of others face to face with her. The proposed pharmacological treatment was risperidone, $1 \mathrm{mg}$ a day.

\section{Schizophrenia patients with no agenesis of corpus callosum (CC group)}

Thirteen young (mean age $24.84 \pm 6.35$ years) patients with schizophrenia (three females), fulfilling the DSM-IV criteria, and with no evident damage or agCC were recruited for this study. The patients were all under antipsychotic medication and were assessed using the PANSS (Kay et al., 1987). They were all treated by the same antipsychotic medication since 15 weeks, quetiapine (mean dosage: $425 \mathrm{mg} /$ day). The mean PANSS total score of the CC group was $88.2(\mathrm{SD}=19.97)$, with $21.11(\mathrm{SD}=6.12)$, and 24.73 $(\mathrm{SD}=9.45)$ on the Positive and Negative scales, respectively. All participants signed an informed detailed consent form approved by the ethics committee prior to participation in the study.

\section{TASK DESCRIPTION AND EXPERIMENTAL DESIGN}

All subjects participated in a passive viewing task during a functional magnetic resonance imaging (fMRI) session in two experimental conditions: Negative and Neutral. During the Negative condition, a series of 44 emotionally laden negative pictures (e.g., plane crash, snake, spider, shark, angry face, sad face, mutilation, accident, burn victim, dead body, etc.) were presented to the participants, whereas in the Neutral condition, they saw a series of 44 emotionally neutral pictures (e.g., tourist, rocks, boat, leaves, outlet, towel, spoon, mug, etc.). The two categories of pictures were selected from the international affective picture system (IAPS; Lang et al., 1988). They were matched as much as possible in terms of visual complexity. The mean valence was $2.66 \pm 1.58$ for the negative pictures and $5.74 \pm 1.47$ for the neutral pictures. The mean arousal level was $6.11 \pm 2.14$ for the negative pictures and $2.97 \pm 2.08$ for the neutral pictures. These mean valence and arousal scores are based on the valence and arousal ratings from Lang's normative groups.

During the functional scan, four blocks of negative and four blocks of neutral images were presented to the subjects in an alternating manner either starting with negative or neutral block, in a counterbalanced manner across subjects. Each image was presented for a period of $2.88 \mathrm{~s}$ and each block-which lasted $31 \mathrm{~s}$ (11 images per block). The blocks were separated by rest periods of $14.4 \mathrm{~s}$, during which subjects viewed a cyan screen. Subjects were instructed to look carefully at each of the 88 pictures presented to them during the run.

\section{IMAGING PARAMETERS}

A 1.5-T whole Body MR System (Magnetom Vision, Siemens, Erlangen, Germany) used normally for clinical purposes at Centre Hospitalier de l'Université de Montréal (CHUM) was 
employed for image acquisition. Prior to the functional run, 165 structural images were acquired in sagittal plane by using an MPRAGE imaging sequence $(\mathrm{TR}=9.7 \mathrm{~ms}$; $\mathrm{TE}=4.0 \mathrm{~ms}$; $\mathrm{FA}=12^{\circ} ; \mathrm{FoV}=250 \mathrm{~mm}^{2}$; matrix size $=256 \times 256$; slice thickness $=0.975 \mathrm{~mm}$, voxel size $=0.975 \mathrm{~mm}^{3} ; 164$ slices $)$. Then, whole brain fMRI was performed using an echo-planar imaging (EPI) sequence measuring blood oxygenation level dependent $(\mathrm{BOLD})$ signal $\left(\mathrm{TR}=3000 \mathrm{~ms} ; \mathrm{TE}=44 \mathrm{~ms} ; \mathrm{FA}=90^{\circ}\right.$; $\mathrm{FoV}=215 \mathrm{~mm}^{2} ;$ matrix size $=64 \times 64$; slice thickness $=5.00 \mathrm{~mm}$, voxel size $=3.36 \mathrm{~mm} \times 3.36 \mathrm{~mm} \times 5 \mathrm{~mm} ; 28$ slices). Functional slices covered the whole brain, were oriented in transverse plane and were angled to be parallel to the AC-PC line. A total of 132 functional volumes were recorded during the functional run.

\section{DATA ANALYSIS}

Brain Voyager QX (Brain Innovation B.V., Maastricht, the Netherlands) software was used for fMRI data preprocessing and analysis. The first two volumes of the functional run, we discarded from the analyses to allow for $\mathrm{T} 1$ equilibration effects. The remaining functional bi-dimensional images of every subject were preprocessed to correct for the difference in time slice acquisition (slice scan time correction). In addition to linear detrending, a high-pass filter of three cycles per time course (frequency domain) was applied to the corrected 2D slices. Then, the functional series was preprocessed to correct for possible motion artifacts in any plane of the tridimensional space and to ensure that the movements in any plane did not exceed $3 \mathrm{~mm}$. These motion corrected functional images were subsequently used to reconstruct the $3 \mathrm{D}$ functional volume for every subject and every run. The $3 \mathrm{D}$ functional volume was aligned with the corresponding 3D anatomical volume, and both were normalized to standard Talairach space (Talairach and Tournoux, 1988). Spatial smoothing using a Gaussian kernel at $8 \mathrm{~mm}$ full width at half maximum (FWHM) was applied to the 3D functional data.

A blocked-design approach was employed for data analysis. Two predictors were defined for the functional run (Negative and Neutral), corresponding to the blocks of presentation of negative, and respective neutral items images. For the group analysis, these predictors were entered as fixed factors in single subject general linear model (GLM), then the parameters of this GLM model were subsequently entered into a second level of analysis corresponding to a random-effect GLM model that was used for group analysis (Penny and Holmes, 2003). The statistical parameters of this latter model were estimated voxelwise for the entire brain and activation maps were computed for the contrast between the two predictors. The criteria used to display the activation maps: a cluster size of 180 adjacent significant voxels $(1 \mathrm{~mm} \times 1 \mathrm{~mm} \times 1 \mathrm{~mm})$ and a statistical threshold for each voxel in the cluster $p<0.005$ (uncorr.). For the analysis of functional data coming from the patient with agCC we performed only difference analysis, using a GLM model with two predictors (Negative and Neutral). To display the activation maps for this individual we use the following criteria: minimal $t$-value $t(127)=4.02 ; p<0.0001$; minimum cluster size 180 voxels.

In the subsequent stage of the analysis, the clusters obtained as a result of various contrasts at the group level were defined as regions of interest (ROIs) and further GLM analyses were performed separately for each of them in order to compare the percentage of signal change at the group level with that in the patient with agCC. These latter analyses were performed taking into account the timecourse of the signal averaged over all voxels in each ROI. The data extracted from Brain Voyager were analyzed with SPSS software (SPSS Inc., Chicago, IL, USA).

\section{RESULTS}

\section{PROCESSING VISUAL INFORMATION}

Contrasting the brain activity during visual blocks of either type (sad or neutral images) with the activity during rest periods (just a fixation cross) revealed a strong activation in left and right occipital poles in both the agCC patient (Figure 2, upper row) as well as in the CC group (Figure 2, lower row). The detailed analysis of the activation clusters revealed that the average individual activation in the CC group was similar to the level of activation of agCC patient in both left [average $t(127)=3.18, p<0.002$, for CC group and $t(127)=3.22, p<0.002$ for agCC patient] and right occipital regions $[t(127)=3.58, p<0.001$, for CC group and $t(127)=4.11$, $p<0.001$ for agCC patient]. Furthermore, the analysis of cerebral activity locked to the onset of visual stimuli (Figure 2, graphs on the right) indicated that the changes in activation were similar in magnitude for both the agCC patient, as well as the for CC patients (about $1 \%$, on average); however, the signal was more variable during the block duration (yellow rectangle) for agCC patient than for the CC group. Finally, the total volume of activation in the whole brain induced by the general processing of visual information was more than 20 times as large in CC group as compared to agCC patient $\left(191.96\right.$ vs. $\left.9.15 \mathrm{~cm}^{3}\right)$. In conclusion, visual cortex activation in response to visual stimuli regardless of their emotional content was comparable in agCC patient and CC group both in terms of localization and intensity of activation; the only difference was that in the CC group a larger area was recruited, which included, among others, subcortical and prefrontal regions.

\section{PROCESSING EMOTIONAL INFORMATION}

When comparing the emotional valence of the visual information ( $\mathrm{SAD}>\mathrm{NEUTRAL}$ ) we observed significant activation in three prefrontal regions, one in temporal and two in cingulate cortex for the CC group (Figure 3, orange clusters) and in a much wider network including cortical and subcortical regions from almost all cerebral lobes for the agCC patient (Figure 3, blue clusters). The laterality index computed separately for each subject for the regions obtained from the same contrast (SAD $>$ NEUTRAL) at the individual level revealed that in the CC group the average laterality was $0.4470(\mathrm{SD}=0.34)$, corresponding to about $44.7 \%$ more voxels activated in one cerebral hemisphere than in the other. In contrast, for the agCC patient, the laterality index was 0.084 , corresponding to only $8 \%$ of lateralization, a value which is more than 1 SD farther away from the CC group average. Regarding the volume of activation for the contrast ( $S A D>$ NEUTRAL) measured at the individual level, we obtained an average of $18.56 \mathrm{~cm}^{3}$ $\left(\mathrm{SD}=27.83 \mathrm{~cm}^{3}\right)$ for the CC group and a value 10 times as large for the agCC patient $\left(189.54 \mathrm{~cm}^{3}\right)$.

In summary, when we assessed the emotional nature of visual information processing we observed a very large, non-specific and non-lateralized cerebral activation in the agCC patient, in contrast with the CC group, which showed a more lateralized and 


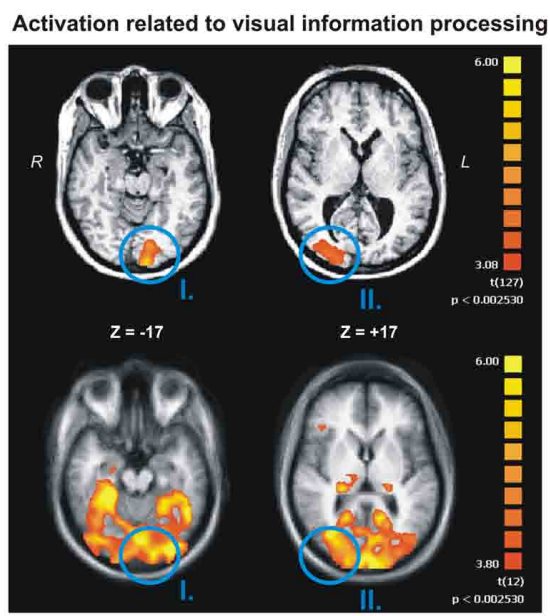

FIGURE 2 |The images to the left present the statistical maps reflecting the activation related to processing of visual information, regardless of the emotional content of the stimuli presented to the participants. The graphs on the left indicate the changes in BOLD signal (percentages) in the occipital poles relative to the onset of the visual
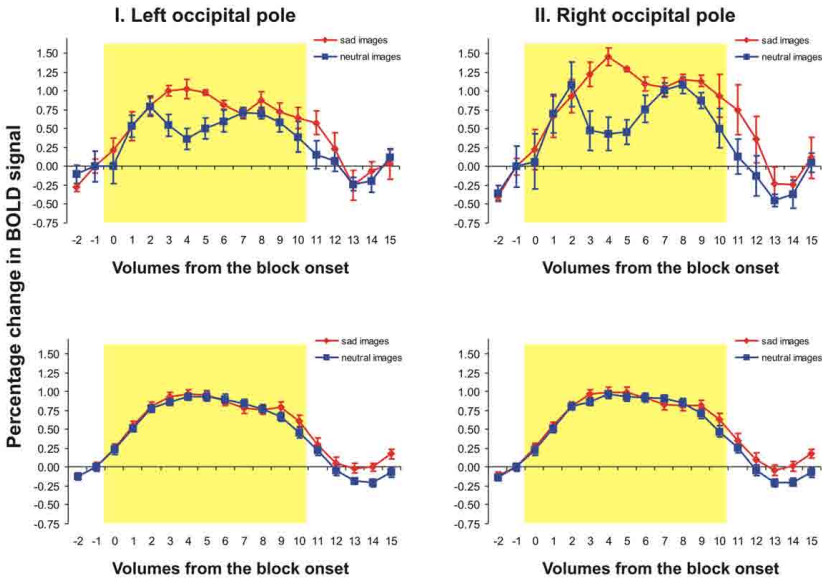

stimulation for emotionally charged (red lines) and neutral (blue lines) stimuli. The upper images and the upper graphs pertain to the schizophrenia patient with CC agenesis, whereas the lower images and graphs reflect the group average (13 schizophrenia patients with no apparent CC agenesis).

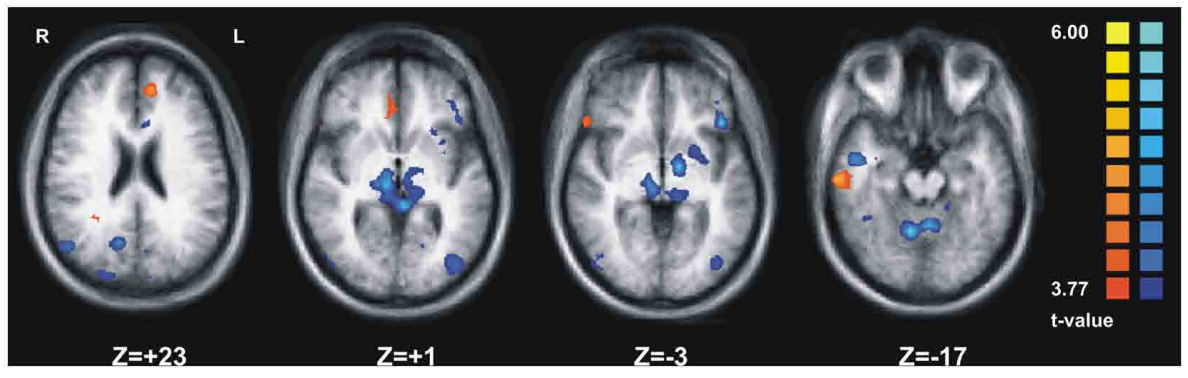

FIGURE 3 |The statistical maps reflecting the brain activation in response to emotional stimuli (contrast: SAD > NEUTRAL). The orange clusters indicate brain activations pertaining to the schizophrenia patient with
CC agenesis, whereas the blue clusters correspond to the activation at the group level for the 13 schizophrenia patients with no apparent CC agenesis (CC group). spatially localized activation. Furthermore, the detailed analysis of the BOLD signal change in each of the six clusters obtained in the CC group revealed that the agCC patient had an opposite activation pattern (Figure 4, red line) relative to most participants with no CC agenesis (Figure 4, green lines).

\section{DISCUSSION}

We assessed here the brain activity in response to emotionally laden visual stimuli in one schizophrenia patient with agCC and in a group of schizophrenia patients of similar age, but with no evident damage to the brain (CC group). The results showed that the visual cortex activation in response to visual stimuli, regardless of their emotional content, was comparable in agCC patient and CC group both in terms of localization and intensity of activation. The only difference in this respect was that in the CC group a larger area was recruited, which included, among others, subcortical and prefrontal regions. In contrast, when comparing the brain activation in response to the emotional charge of the visual stimulation we observed a very large, non-specific and non-lateralized cerebral activation in the agCC patient, in contrast with the CC group, which showed a more lateralized and spatially localized activation. The analysis of the BOLD signal change in each of the activated regions obtained in the CC group revealed that the agCC patient actually had an opposite activation pattern relative to most participants with no CC agenesis, indicating a dysfunctional response to these kind of stimuli, consistent with the clinical presentation of this particular patient.

The congenital absence of CC, agenesis, is the main "natural" pathology of this brain structure and it has recently been estimated to have a prevalence of 1:4000 (Paul et al., 2007). While its etiology is yet unclear, there are studies linking this condition to genetic and environmental factors occurring prenatally, typically between the $3 \mathrm{rd}$ and 12 th week of pregnancy when this structure develops. For instance, both in animal models, as well as in humans, the pioneer axons from cingulate cortex are the first to cross the midline during embryonic development, providing guidance, and support for the 


\section{Brain activation for each participant in the two experimental conditions in regions activated significantly by the contrast SAD>NEUTRAL for CC group}
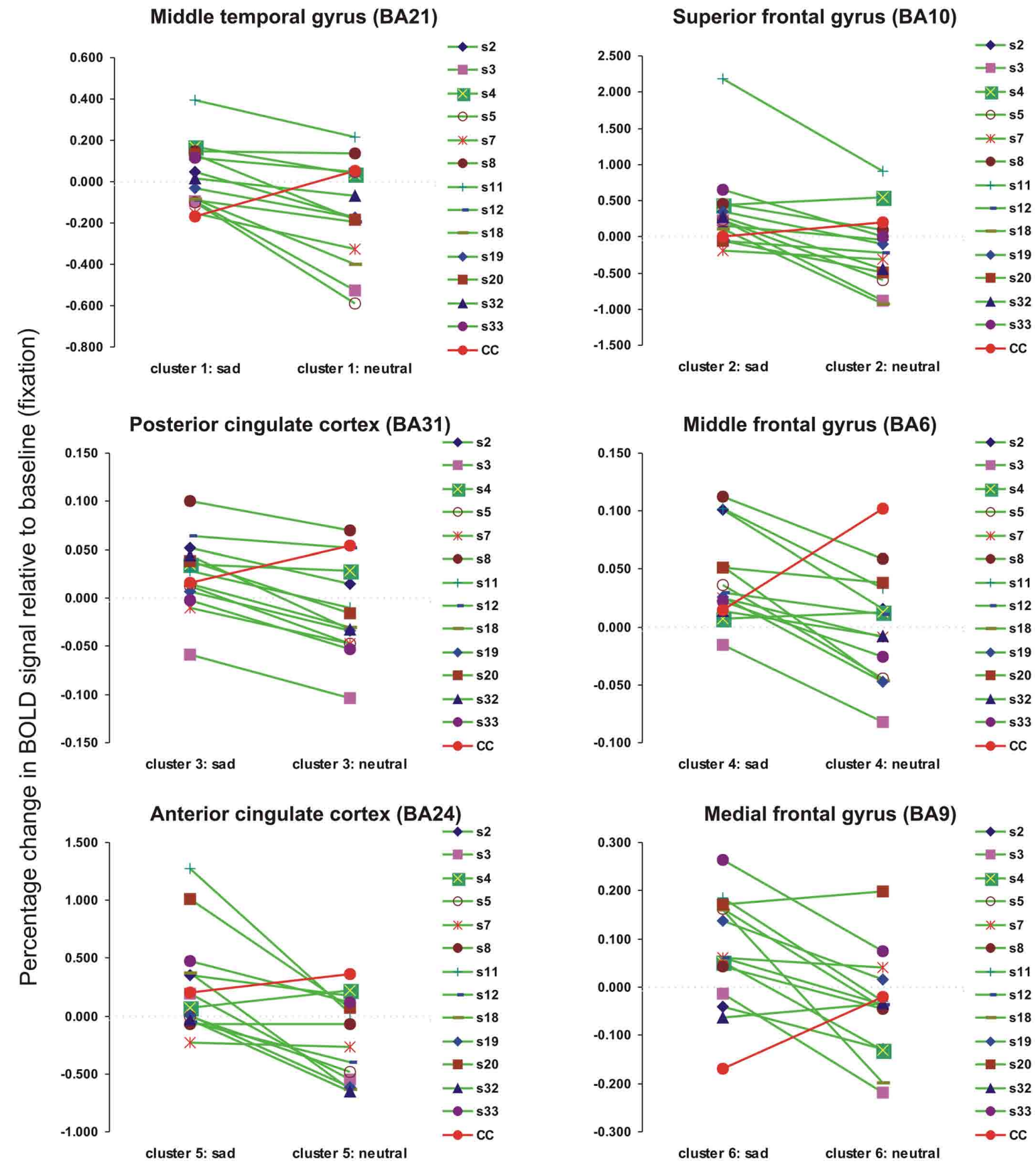
factors, infectious, vascular, and toxic causes of agCC were also identified (Paul et al., 2007).

Based on the topography of the agenesis in our case study, which encompassed the dorsal CC aspect, above the genu, and surrounding the midline, as well as the istmus and anterior half of the spelnium, there are a number of cortical areas that may be disconnected from their contralateral homotopic parts. Parts of dorsolateral prefrontal cortex (BA9/BA44/BA45), including Broca's area, as well as the superior temporal cortex, including Wernicke's area, are usually connected to parts of CC that are absent in our patient. Even though the patient did not show marked language problems, she had nevertheless difficulty in encoding and interpreting verbal material. Other cortical regions that could be disconnected in our clinical case are: anterior cingulate cortex (BA32), posterior parietal cortex (BA7), supplementary motor, and premotor areas (BA6). Taken together, these regions are part of mirror neuron system (Iacoboni and Mazziotta, 2007) and the clinical evidence that they could be affected by the agCC in our patient is given by her difficulty in imitating the other's gestures.

Our imaging results illustrate two main points. On the one hand, we showed that in both our agCC patient and in the group of patients with no apparent damage to the CC, there was similar brain activity during basic visual information processing. Corroborated with the neurological evaluation showing that the patient did not have difficulty integrating visual information from the two visual fields, this indicates that, contrary to cases of corpus callosotomy (Schulte and Muller-Oehring, 2011), the developmental dysgenesis of this structure does not affect the visual pathways and the integration of information from both visual fields. This may be due to the fact that the posterior part of the splenium, which connects the occipital and inferior temporal cortices, was intact in our agCC patient. On the other hand, the CC group and agCC patient showed different patterns of brain activity when the emotional nature of visual information was taken into account. Corroborated with the presence of visual distortions and hallucinations, as well as with the increased suspicion and persecution beliefs in agCC patient, our findings suggest that the CC agenesis may be a compounding factor in exacerbating some of the positive symptoms in schizophrenia. Of course, further research is necessary to actually link the severity of the positive symptoms

\section{REFERENCES}

Arnone, D., McIntosh, A. M., Tan, G. M., and Ebmeier, K. P. (2008). Meta-analysis of magnetic resonance imaging studies of the corpus callosum in schizophrenia. Schizophr. Res. 101, 124-132.

Bigelow, L. B., Nasrallah, H. A., and Rauscher, F. P. (1983). Corpus callosum thickness in chronic schizophrenia. Br. J. Psychiatry 142, 284-287.

Booth, R., Wallace, G. L., and Happe, F. (2011). Connectivity and the corpus callosum in autism spectrum conditions Insights from comparison of autism and callosal agenesis. Prog. Brain Res. 189, 303-317.
Brown, W. S., and Paul, L. K. (2000). Cognitive and psychosocial deficits in agenesis of the corpus callosum with normal intelligence. Cogn. Neuropsychiatry 5, 135-157.

Buklina, S. B. (2005). The corpus callosum, interhemisphere interactions, and the function of the right hemisphere of the brain. Neurosci. Behav. Physiol. 35, 473-480.

Clapcote, S. J., and Roder, J. C. (2006). Deletion polymorphism of Disc1 is common to all 129 mouse substrains: implications for genetargeting studies of brain function. Genetics 173, 2407-2410.

to structural damage of the CC, but in the future it may be an important indicator for clinicians to suspect callosal damage when positive symptoms are very prominent in the early onset of the disease.

\section{LIMITATIONS}

There are two main limitations in our study. First, is that we have only one case with CC agenesis and schizophrenia; it would have been better to compare two groups instead of a case with a group. However, given the rarity of the agenesis (1:4000) in the general population, finding enough cases is very difficult. Another limitation in our study is the lack of a clinical case that presents CC agenesis, but does not have schizophrenia. Such a case would have been useful in illustrating the impact of the structural damage on the emotional information processing in the absence of schizophrenia. However, we believe that despite these limitation, our data can be useful in showing the compounding effect of a structural damage in schizophrenia on the brain functioning.

\section{CONCLUSION}

Our case study shows a patient who has a dual diagnosis: schizophrenia and corpus callosum agenesis. We know that the disconnection hypothesis suggests that the core symptoms of schizophrenia are related to aberrant connectivity between distinct brain areas. Both functional and structural neuroimaging studies have been conducted to investigate this hypothesis, across the full course of the disorder; from people at ultra-high-risk of developing psychosis to patients with established schizophrenia. Our case study is a first-episode psychosis leading to a schizophrenia and it was compared to a group a young schizophrenia patients. The role of the CC in the emotional information processing confirms the needed connectivity in the two hemispheres since the observation of a very large, non-specific and non-lateralized cerebral activation in the agCC patient, in contrast with the CC group, which showed a more lateralized and spatially localized activation.

\section{ACKNOWLEDGMENTS}

Dr. Emmanuel Stip is the Eli Lilly Canadian Chair in Schizophrenia. This study was conducted by Emmanuel Stip as a clinical scientist Junior2, funded by the FRSQ and as part of investigator initiated trials funded by Astra Zeneca Canada.

de Lacoste, M. C., Kirkpatrick, J. B. and Ross, E. D. (1985). Topography of the human corpus callosum. J. Neuropathol. Exp. Neurol. 44, 578-591.

Hallak, J. E., Crippa, J. A., Pinto, J. P., Machado de Sousa, J. P., Trzesniak, C., Dursun, S. M., McGuire, P., Deakin, J. F., and Zuardi, A. W. (2007). Total agenesis of the corpus callosum in a patient with childhood-onset schizophrenia. Arq. Neuropsiquiatr. 65 1216-1219.

Highley, J. R., Esiri, M. M., McDonald, B., Cortina-Borja, M., Herron, B. M., and Crow, T. J. (1999). The size and fibre composition of the corpus callosum with respect to gender and schizophrenia: a post-mortem study. Brain 122(Pt 1), 99-110.

Iacoboni, M., and Mazziotta, J. C. (2007). Mirror neuron system: basic findings and clinical applications. Ann. Neurol. 62, 213-218.

Kay, S. R., Fiszbein, A., and Opler, L. A. (1987). The positive and negative syndrome scale (PANSS) for schizophrenia. Schizophr. Bull. 13, 261-276.

Lang, P. J., Öhman, A., and Vaitl, D. (1988). The International Affective Picture System (Photographic Slides). Gainesville: Center for Research in Psychophysiology, University of Florida. 
Micoulaud-Franchi, J. A., Bat-Pitault, F., Da Fonseca, D., and Rufo, M. (2011). Early onset schizophrenia and partial agenesis of corpus callosum. Arch. Pediatr. 18, 189-192.

Paul, L. K., Brown, W. S., Adolphs, R., Tyszka, J. M., Richards, L. J., Mukherjee, P., and Sherr, E. H. (2007). Agenesis of the corpus callosum: genetic, developmental and functional aspects of connectivity. Nat. Rev. Neurosci. 8, 287-299.

Paul, L. K., Lautzenhiser, A., Brown, W. S., Hart, A., Neumann, D., Spezio, M., and Adolphs, R. (2006). Emotional arousal in agenesis of the corpus callosum. Int. J. Psychophysiol. 61, 47-56.

Penny, W. D., and Holmes, A. J. (2003). "Random-effects analysis," in Human Brain Function, eds R. S. Frackowiak, K. J. Friston, C. Frith, R. Dolan, C. J. Price, S. Zeki, J.
Ashburner, and W. D. Penny (London: Academic Press), 843-850.

Rash, B. G., and Richards, L. J. (2001). A role for cingulate pioneering axons in the development of the corpus callosum. J. Comp. Neurol. 434, 147-157.

Ren, T., Anderson, A., Shen, W. B., Huang, H., Plachez, C., Zhang, J., Mori, S., Kinsman, S. L., and Richards, L. J. (2006). Imaging, anatomical, and molecular analysis of callosal formation in the developing human fetal brain. Anat. Rec. A Discov. Mol. Cell. Evol. Biol. 288, 191-204.

Rosenthal, R., and Bigelow, L. B. (1972). Quantitative brain measurements in chronic schizophrenia. Br. J. Psychiatry 121, 259-264.

Schulte, T., and Muller-Oehring, E. M. (2011). Contribution of callosal connections to the interhemispheric integration of visuomotor and cognitive processes. Neuropsychol. Rev. 20, 174-190.

Symington, S. H., Paul, L. K., Symington, M. F., Ono, M., and Brown, W. S. (2011). Social cognition in individuals with agenesis of the corpus callosum. Soc. Neurosci. 5, 296-308.

Talairach, J., and Tournoux, P. (1988). Co-planar Stereotaxic Atlas of the Human Brain. New York: Thieme Medical Publishers.

Tamietto, M., Adenzato, M., Geminiani, G., and de Gelder, B. (2007). Fast recognition of social emotions takes the whole brain: interhemispheric cooperation in the absence of cerebral asymmetry. Neuropsychologia 45, 836-843.

Wechsler, D. (2008). WAIS-IV Manual. New York: NY: The Psychological Corporation.

Conflict of Interest Statement: The authors declare that the research was conducted in the absence of any commercial or financial relationships that could be construed as a potential conflict of interest.

Received: 31 August 2011; accepted: 04 January 2012; published online: 03 February 2012.

Citation: Lungu $O$ and Stip E (2012) Agenesis of corpus callosum and emotional information processing in schizophrenia. Front. Psychiatry 3:1. doi: 10.3389/fpsyt.2012.00001

This article was submitted to Frontiers in Neuropsychiatric Imaging and Stimulation, a specialty of Frontiers in Psychiatry. Copyright (c) 2012 Lungu and Stip. This is an open-access article distributed under the terms of the Creative Commons Attribution Non Commercial License, which permits non-commercial use, distribution, and reproduction in other forums, provided the original authors and source are credited. 